Pavle Sekeruš*

Ivana Živančević Sekeruš

Virdjinija Popović

Faculté de Philosophie et Lettres,

Université de Novi Sad
UDK: 17.023.36(497.11:44)

821.133.1:821.163.41 Kiš D.

DOI: $10.19090 /$ gff.2021.1.93-104

Originalni naučni rad

\title{
PARIS EST UNE FOIRE AUX ENCHERES LITTERAIRE ${ }^{* *}$
}

\author{
Danilo Kiš comme médiateur culturel entre la France et la Serbie
}

Prosateur subtile qui se rêvait poète, intellectuel libre et farouchement indépendant, Danilo Kiš est en contact avec la culture française depuis son enfance. Il apprend le français à l'école et commence à publier ses premières traductions du français pendant ses études à Belgrade. Engagé comme lecteur de langue serbo-croate, il séjourne à Strasbourg, Bordeaux, et Lille pour s'installer définitivement à Paris où il vit jusqu'à sa mort. Ses œuvres les plus importantes écrites en France, traduites et publiées dans les plus grandes maisons d'édition parisiennes, laisseront leur marque sur la culture française et mondiale. Par son activité, Danilo Kiš s'impose comme un point central d'échange entre deux espaces culturels: le français, qui lui ouvre la porte vers le monde et l'espace serbe, yougoslave et centre-européen. Le premier lui offre la tolérance de la ville cosmopolite et la liberté, mais aussi la politisation insupportable et la prétention inhérente au grand centre et le deuxième, la chaleur de la langue maternelle, la proximité et la sécurité, mais aussi l'environnement provincial borné, l'anarchie et la terreur politique. Combinant la théorie des transferts culturels de Michel Espagne et les théories de Pascale Casanova sur la « littérature mondiale » qui supposent l'analyse des espaces culturels transnationaux, on découvre Kiš comme médiateur culturel entre deux mondes.

Mots clés : Danilo Kiš, transfert culturel, France, Serbie, auteur et traducteur

\footnotetext{
*psekerus@ff.uns.ac.rs

${ }^{* *}$ L'article est le résultat du travail dans le cadre du projet Transferts culturels et champs intellectuels transnationaux. Modernité et antimodernité à l'Ouest et à l'Est: France, Roumanie, Serbie, nr. 21-AUF/01.03.2019 (Kulturni transferi $i$ transnacionalna intelektualna polja. Modernost $i$ antimodernost na Zapadu i na Istoku: Francuska Rumunija, Srbija, no 21-AUF/01.03.2019). Il a été présenté au colloque à Novi Sad le 19 novembre 2019.
} 
Danilo Kiš est né le 22 février 1935 à Subotica, dans le Royaume de Yougoslavie, ville située près de la frontière hongroise et rattachée, depuis la fin de la Première guerre mondiale, à ce nouvel état avec l'ensemble du territoire appelé Voïvodine. Son père est un juif hongrois Edouard Kohn qui, dans une tentative désespérée d'assimilation et de mimétisme dans un climat antisémite en Hongrie, change son nom d'origine en Kiš, qui est commun en Hongrie. Sa mère est Milica Dragićević, monténégrine orthodoxe de Cetinje. La famille déménage à Novi Sad aussitôt après la naissance de Danilo. Avec le même souci de préservation, ses parents baptisent Danilo et sa sœur en 1939 dans une église orthodoxe.

Il vit à Novi Sad jusqu'à l'âge de sept ans. La Deuxième guerre mondiale éclate. Les fascistes hongrois occupent la ville et en hiver 1942 massacrent les Juifs et les Serbes en les jetant dans le Danube, etant alors glacé. La famille Kiš se retire dans le village natal de son père en Hongrie, où Danilo continue sa scolarité. La tragédie juive de Novi Sad se double d'une tragédie familiale : le père est emmené à Auschwitz en 1944 où ils perdent alors toute trace de lui.

A partir de 1947, Danilo vit chez son grand père maternel à Cetinje au Monténégro. Il quitte Cetinje en 1954 pour s'inscrire à l'Université de Belgrade où il termine ses études de littérature générale et comparée en 1958 (Janičić \& Lazić, 1999).

Présent dans la ville intellectuelle de la capitale, il n'échappe pas au climat francophile qui règne à Belgrade. Bien que la chute de la famille royale des Karadjordjević et la création d'un état socialiste après la Deuxième guerre mondiale coupent les relations étroites que le royaume avait avec la France, la brouille de Tito avec Staline tourne les regards de Belgrade à nouveau vers l'Occident dans les années cinquante. Danilo parle le hongrois, le russe et le français mais la plupart de ses traductions sont faites à partir d'œuvres d'auteurs français. La première publiée est de 1957, et c'est une étude sur Proust de Pierre de Boisdeffre et la dernière, publiée en 1989, l'année de sa mort, le livre de Jacques Rossi Le manuel du Goulag. Entre ses deux dates il traduira tout un pan de la littérature française: Ronsard, Malherbe, Corneille, Molière, Voltaire, Sartre, Baudelaire, Prévert, Queneau, Verlaine, Lautréamont, Butor, Michaux, Henry-Lévy, pour ne citer que quelques-uns.

De nombreuses traductions de poésies témoignent de ses propres aspirations poétiques, jamais satisfaites en profondeur. Il racontait qu'il traduisait les poètes qui disaient mieux que lui ses propres obsessions et questionnements, pour conclure que cela est d'ailleurs la raison pour laquelle il renonce à la carrière de poète et devient alors prosateur (Stojanović, 1999). 
La traduction du français fera partie de ses travaux littéraires jusqu'à la fin de sa vie et jouera un rôle important dans le contexte du transfert culturel francoserbe....

Pascale Casanova ${ }^{1}$ voit justement la traduction comme une activité hiérarchisée selon les rapports de force entre les champs littéraires nationaux. Les inégalités et les hiérarchies, tant littéraires que linguistiques, qui ordonnent le champ littéraire mondial, transforment la traduction en «échange inégal », loin des échanges horizontaux et transferts amicaux, des moyens de faire passer simplement les textes d'un champ littéraire national à un autre, comme elle est souvent décrite. Comprise ainsi, la traduction empêche de repérer et de comprendre les enjeux réels de la circulation internationale des textes littéraires (Casanova, 2002).

D'après Casanova les inégalités sont le résultat du capital linguisticolittéraire spécifique d'une langue qui dépend du nombre de polyglottes littéraires qui la pratiquent et du nombre de traducteurs littéraires - tant à l'importation qu'à l'exportation - qui font circuler les textes depuis ou vers cette langue littéraire.

La distribution inégale de ce capital ordonne le champ linguistico-littéraire selon une opposition entre les langues littéraires dominées (langues récemment « nationalisées » c'est-à-dire devenues langues nationales relativement tardivement, dotées de peu de capital littéraire, de peu de reconnaissance internationale, et d'autre part les langues dominantes, qui, du fait de leur prestige spécifique, de leur ancienneté, du nombre de textes déclarés universels écrits dans ces langues, sont dotées d'un volume important de capital littéraire.

Casanova divise les langues dominées en quatre groupes distincts où les langues orales, dont l'écriture a été récemment fixée forment le premier, dont le capital linguistico littéraire est le plus faible. Le quatrième est composé des langues de grande diffusion comme l'arabe, le chinois ou l'hindi qui, bien que dotées de grandes traditions littéraires, restent peu connues sur le marché littéraire international. Nous avons reconnu le troisième groupe de langue dominées comme celui de la langue serbe : "Les langues de culture ou de tradition ancienne liées à de 'petits' pays, comme le néerlandais ou le danois, le grec ou le persan... [...] Elles ont

\footnotetext{
${ }^{1}$ Pascale Casanova (1959-2018) est chercheuse et critique littéraire française. Elle est connue en particulier pour sa thèse sur la «République mondiale des lettres ». Ses recherches portent sur la constitution du champ littéraire international et l'analyse des textes littéraires en tant que positions et objets de lutte dans l'espace mondial.
} 
une histoire et un crédit relativement importants, mais peu de locuteurs, sont peu pratiquées par les polyglottes et sont peu reconnues en dehors des frontières nationales. » (Casanova, $2002:$ 9).

Casanova désigne une stratégie pour les écrivains issus des champs littéraires nationaux dominés : « ... s'ils veulent entrer dans la concurrence littéraire mondiale, [ils doivent] travailler à importer du capital, à gagner de l'ancienneté et de la noblesse en «nationalisant » (c'est-à-dire ici, très précisément, en traduisant dans la langue nationale) les grands textes universels, soit ceux qui sont reconnus comme capital universel dans l'univers littéraire.»(Casanova, 2002: 10). C'est ce que Casanova appelle le « détournement de capital » et c'est justement cela que, selon cette conception, faisait Danilo Kiš en traduisant des textes des classiques tout court et des classiques de la modernité de la littérature française. Mais comme les traducteurs sont souvent auteurs aussi, les traductions se transforment souvent en instruments de bataille privilégiés des écrivains les plus individualistes et autonomes, car elles leur permettent de rompre avec les normes de leur espace national et d'introduire des procédés littéraires consacrés dans les centres littéraires internationaux.

Danilo Kiš, a traduit ainsi en serbo-croate les poètes hongrois Petôfi, Ady, Radnotti, Atilla József, les russes Mandelstam, Essenine, Marina Tsvetaieva, ainsi que les Français Lautréamont, Prévert, Queneau, etc. Comme les autres traducteurs, il importait la modernité décrétée aux centres littéraires internationaux et la faisait connaître dans son champ national. Par cette activité il jouait un rôle essentiel dans le processus d'unification du champ littéraire mondial, conclut Casanova.

Parallèlement avec ce travail de traduction, l'auteur qui se rêvait poète ${ }^{2}$, publie ses premiers textes en prose : en 1962 apparaissent deux courts romans - $L a$ Mansarde (Mansarda) et Psaume 44 (Psalm 44) - qui découvrent déjà les traits majeurs de sa poétique, l'autobiographie et le jeu avec la documentation, les procédés si typiques pour le postmodernisme. Détectés d'abord dans l'œuvre de l'auteur argentin Jorge Louis Borges, qui est traduit pour la première fois en français en 1951, et ainsi visibles dans une langue dominante, ces procédés sont

\footnotetext{
${ }^{2}$ Dans un interview avec Bernard Pivot il declare : ... je savais que je serais un écrivain mais je pensais devenir un poète. Et je me suis préparé pour être poète. J'ai commencé donc par la traduction de la poésie comme une sorte de préparation pour le métier, le difficile métier de poète. Finalement j'ai épuisé mes forces poétiques en faisant des traductions de poètes hongrois, russes et français (Pivot, 2013).
} 
entrés dans le processus interminable de la circulation des modèles littéraires. Modifiés dans les œuvres des auteurs comme Kiš, en fonction de leurs poétiques personnelles et des contextes culturels, ils sont, à leur tour, offerts comme modèles à suivre.

Le travail romanesque de Kiš continue avec la trilogie familiale composée d'un recueil de courts récits intitulé Chagrins précoces (Rani jadi, 1969) et de deux romans : Jardin, cendre (Bašta, pepeo, 1965) et Sablier (Peščanik, 1972). Dans ces trois livres le héros principal est le père de l'auteur, Edouard Sam, tragiquement disparu à Auschwitz : " tantôt comme une curieuse symbiose de Charlot et de Don Quichotte, tantôt comme un mélange étrange de Meistersinger, d'Ahasvérus et d'un philosophe itinérant, avant de se transformer finalement, dans [le roman] Sablier, en ce qu'il était en réalité : un homme fragile, traqué, broyé par les fureurs de l'Histoire » (Srebro, 1980).

À partir des années 1960, Danilo Kiš est engagé comme lecteur de serbocroate dans plusieurs universités en France : à Strasbourg (1962-1964), à Bordeaux (1973-1976) et à Lille (1979-1985). C'est à Strasbourg qu'il écrit le roman Jardin, cendre, recueil de sept nouvelles que certains appellent roman. Un tombeau pour Boris Davidovitch nait à Bordeaux, et Encyclopédie des morts, un recueil de neuf nouvelles, à Paris (Keiflin, Marković, Stanić, 2014 : 6). Il écrit en serbo-croate et publie ses œuvres d'abord en Yougoslavie, pour les faire traduire et publier plus tard en France. A partir de 1979, il s'installe définitivement à Paris, et passe la dernière décennie de sa vie dans cette ville où il meurt le 15 octobre 1989.

La décision de s'installer définitivement à Paris est prise suite à des évènements produits par la publication à Belgrade et à Zagreb, en 1976, du roman Un tombeau pour Boris Davidovitch, qui a déclenché une des plus grande polémique de la littérature yougoslave. Des centaines de pages de livres et de textes critiques dans des journaux et des revues, pour et contre ce petit bouquin, faisaient trembler le monde littéraire yougoslave pendant plusieurs années. Sur le site de Serbica Milivoj Srebro résume la polémique de la façon suivante :

Dans ce livre qui a pour sujet la répression stalinienne en URSS, l'écrivain nous mène, à travers ses sept nouvelles comme à travers sept cercles dantesques, dans un monde d'horreur fondé sur une idéologie qui, tout en promettant le paradis sur terre, a finalement offert l'enfer. Mais, ce livre a plus choqué les esprits traditionalistes par les procédés littéraires choisis par l'écrivain que par son sujet, procédés qui démontrent brillamment que toute vérité officielle est sujette à caution. Tout en usant de la technique de l'apparence savante - références, notes, citations - Kiš réalise une symbiose jusque-là inouie, symbiose de documents 
authentiques et d'apocryphes, de faux et de vrais témoignages, qui mêle sans vergogne les destins de personnages réels et fictifs. Ces procédés osés et insolites, expressions d'une autre conception de l'écriture et d'une autre approche de la réalité, différente et originale, n'ont pas pu, évidemment, passer inaperçus : ce sont donc eux d'abord, qui ont heurté de plein fouet les esprits au goût conservateur, tout en provoquant une vive polémique, qui marquera profondément la vie intellectuelle et littéraire à Belgrade, dans la deuxième moitié des années soixantedix du 20e siècle. (Srebro, 1980).

Derrière cette appellation « esprits traditionalistes » se trouvent des groupes et des individus disparates: communistes dures qui ne supportaient pas la discussion sur les crimes staliniens ${ }^{3}$, les nationalistes qui ne voyaient pas d'un bon œil son yougoslavisme ${ }^{4}$, son mépris du nationalisme et son individualisme, le refus de choisir son camp, aussi bien que des jaloux de ses succès littéraires à l'étranger. Son ami belgradois, Filip David parle même d'une forme d'antisémitisme non avoué.

«Jer za intelektualce ovoga veka, ovog našeg doba, postoji samo jedan ispit savesti, postoje samo dva predmeta iz kojih se padne ne na godinu, nego zbog kojih se gubi pravo (moralnog) glasa jednom zauvek: fašizam i staljinizam. Sve ostalo su trice i kučine », napisao je 1979. Danilo Kiš. Kako to da je poslednjih dvadeset i pet godina veliki broj «intelektualaca ovog veka» $\mathrm{u}$ svim jugoslovenskim republikama nepovratno pao na «ispitu » o kome govori Danilo Kiš? (Nikčević, 2014).

[Traduction : Car pour les intellectuels de ce siècle, de notre époque, il n'y a qu'un seul test de conscience, il n'y a que deux sujets qui ne nous font pas perdre une année de nos études, mais qui nous font perdre le droit de vote (moral) une fois pour toutes: le fascisme et le stalinisme. Tout le reste n'est que de petites choses », écrivait Danilo Kiš en 1979. Comment se fait-il qu'au cours des vingt-cinq

\footnotetext{
${ }^{3}$ Sur la page WEB de plateforme Kaleidoscop de 2016 sous le titre «L'étérnité du Tombeau de Kiš » Jelena Pavlović écrit : «Les attaques contre le stalinisme ont attiré également l'attention de l'homme d'Etat russe Leonid Brezhnew qui se plaignait à Tito pendant une de ses visites en Yougoslavie d'un écrivain yougoslave antisoviétique ce qui a provoqué l'officialisation et le commencement de la campagne antikisienne.» Difficile à vérifier ces déclaration mais nous devons ajouter que Brezhnew a visité la Yougoslavie au novembre 1976 et que le livre de Kiš etait publié au mois de juin de la même année.

${ }^{4}$ Il tenait beaucoup à son identité yougoslave en se définissant comme «le seul écrivain yougoslave sur cette planète » (Kiš, 1995 : 274) .
} 
dernières années, un grand nombre «d'intellectuels de ce siècle » dans toutes les républiques yougoslaves aient irréversiblement échoué à «l'examen » dont parle Danilo Kiš?]

Parmi les crimes qui lui sont reprochés le principal se résume en un mot : le plagiat. Ces détracteurs attaquent son procédé d'ars combinatoria, vieille technique poétique qui implique l'interpolation du matérielle documentaire et fictif, mêlant les citations cachés et référencés dans un nouveau contexte du texte littéraire. Pour justifier ses procédés et pour attaquer ses détracteurs, Danilo Kiš publie en 1978 le livre La leçon d'anatomie, qui représente son crédo poétique, le crédo d'une poétique que certains appellent postmoderne.

Le procès judiciaire contre ses détracteurs gagné, les prix littéraires, la reconnaissance et le soutient des critiques et du public ne suffisent pas à calmer l'auteur blessé pour qui l'atmosphère à Belgrade devient invivable. Il s'auto exile définitivement à Paris où il reste jusqu'à sa mort précoce, à l'âge de 54 ans.

La relation avec les milieux intellectuels parisiens est ambivalente, parfois même difficile. D'un côté il y avait la tolérance de la ville cosmopolite et la liberté d'un auteur relativement inconnu, mais de l'autre on trouve la politisation insupportable « la sartrose » (Kiš, 1993 : 49) comme il l'appelait et la prétention et le snobisme du grand centre. Il s'en plaindra dans une interview avec Janine Matillon intitulée Qu'est ce qu'un écrivain yougoslave à Paris? (Matillon, 1980). Il se dit choqué par le gauchisme de l'intelligentsia parisienne, par les «courbettes faites à Moscou » et ensuite à Mao, par tous ceux qui ne croyaient plus au «bilan globalement positif $»^{5}$ :

C'est à tout ça que je pensais à Belgrade, à la crédulité des Français (je tire mon chapeau aux exceptions), à ce rêve d'un monde nouveau et meilleur, dans lequel ils ronflent comme dans un bon lit, à toutes les illusions qu'ils s'entêtent à fabriquer, en dépit des faits, en dépit de tout. À leur monde en noir et blanc, ici c'est le Bien, ici c'est le Mal, là c'est la Gauche, là c'est la Droite, ils s'accrochent à cet équilibre (Matillon, 1980).

\footnotetext{
5 Dans un article dans L'Humanité du 13 février 1979, préparatoire au XXIII ${ }^{\mathrm{e}}$ congrès du parti, George Marchais en parlant des pays de l'Est dit «Tous ces éléments témoignent de la supériorité du système social nouveau que se sont donné les pays socialistes (...), nous les avons en vue lorsque nous apprécions leur bilan comme globalement positif.»
} 
Avec cela il y a aussi «Paris centre du monde », une constatation prononcé avec une nuance ironique et une certaine amertume.

Vous voyez, « de ce qu'on appelle »... la culture française, pour moi, aujourd'hui, c'est d'abord une culture de médiation, un sol, si vous voulez, où peuvent fleurir, enfin je veux dire pousser, une culture européenne et même une culture universelle. Pour être plus précis : il me semble que Paris est toujours, et de plus en plus, une vraie foire, vous savez, une foire aux enchères, où l'on vend aux enchères tout ce que le monde de la culture a craché ailleurs, sous d'autres méridiens... Il faut passer par Paris pour exister. La littérature hispano-américaine, la grande littérature hispano-américaine, elle a existé avant les Français, comme l'existentialisme, le formalisme russe, etc., etc., mais pour être élevée au rang de patrimoine universel, pour devenir le Bien de la terre entière, il a fallu qu'elle passe par Paris. Voilà à quoi ça sert, la cuisine parisienne. Émigrations, universités, thèses et thèmes, traductions, explications : la cuisine, quoi (Matillon, 1980).

Ces phrases sont comme un écho de Pascal Casanova qui disait que l'inégalité linguistico-littéraire fait que la valeur littéraire d'un texte sur le marché littéraire dépend de la langue dans laquelle il est écrit. Cette inégalité a des effets si puissants qu'elle peut empêcher la reconnaissance ou la consécration des auteurs qui pratiquent des langues dominées. Ces langues deviennent ainsi une sorte de cage et de prison linguistique pour les auteurs. Pour un écrivain de langue dominée, la bataille pour la traduction, doit permettre l'accès aux centres, «aux instances critiques et consécratrices ", la possibilité de montrer son œuvre à ceux qui décrètent ce qu'il faut lire. Loin d'être une simple naturalisation, la traduction en langue dominante est «l' obtention d'un certificat de littérarité » (Casanova, 2002 : 14).

Et Danilo Kiš est passé par la traduction en français qui lui a permis la reconnaissance de la critique française et mondiale. Il est l'auteur yougoslave le plus traduit après le prix Nobel de 1961, Ivo Andrić (Srebro, Novi Sad : 753). Dans l'article Danilo Kiš en France, "Exil », création, réception, 1935-1989, Keiflin, Marković et Stanić soulignent qu'à partir de 1970 deux grandes maisons d'éditions, Gallimard et Fayard, s'occupent de la publication de ses œuvres. Il est publié dans la collection «Du monde entier» chez Gallimard et la collection «Littérature étrangère » chez Fayard avec les auteurs comme Roth, Fuentes, Vargas Llosa, Handke, Brodsky, Soljenitsyne, Nabokov. Il est reconnu ainsi comme auteur européen et mondial.

Si la réception de son œuvre reste relativement faible dans les années 70, les années 80 marquent l'essor international de sa littérature. La publication du livre 
Un tombeau pour Boris Davidovitch en 1979, trois ans après l'édition serbo-croate, est un tournant. Dans le climat encore chaud de discussions animées qui ont suivi la publication française de l'Archipel Goulag de Soljenitsyn (1973), le livre de Danilo Kiš remet du sel sur la plaie d'une certaine gauche française qui refusait catégoriquement de lire Soljenitsyne pour ne pas être obliger de remettre en question ses conceptions du bien et du mal: «l'Est est le paradis, l'Occident est l'enfer » (Kiš, $1995: 122)$.

En contact avec les étudiants des universités françaises, Kiš dit avoir découvert que, lorsqu'il raconte une histoire intéressante, il est écouté, peu importe l'idée derrière l'anecdote :

[...] au début, le monde refusa d'admettre la terrible réalité des camps soviétiques — dont l'existence est un des faits cruciaux de ce siècle —, raison pour laquelle les intellectuels de gauche refusèrent même de lire ce livre, L'Archipel du Goulag, sous prétexte qu'il était le fruit d'un sabotage idéologique et d'un complot de la droite. Comme il était impossible, donc, de discuter avec ces gens sur le plan des idées générales, car ils avaient des opinions a priori et agressives, je me suis vu contraint de développer mes arguments sous forme d'anecdotes et d'histoires, en me basant sur ce même Soljenitsyne, ainsi que sur Stajner, les Guinzbourg, Nadejda Mandelstam, Medvedev, etc. Ces anecdotes étaient la seule forme de discussion acceptable pour eux, c'est-à-dire qu'ils écoutaient, à défaut de comprendre. (Ibid., p. 123).

La Nouvelle revue française et La Quinzaine littéraire sont parmi les revues qui contribuent le plus à ce que sa littérature, surtout après la publication de Boris Davidovitch, soit appréciée en France. Les périodiques comme Le Figaro, Le Nouvel Observateur, la Libération suivent. Les critiques français reconnaissent dans l'œuvre de Kiš l'atmosphère de l'Europe centrale, sa Pannonie, cette identité et cette culture forgées à l'époque de l'empire Austro-hongrois, où le multilinguisme, le multiconfessionalisme et la diversité ethnoculturelle sont la règle. Ce monde laissé à l'oubli derrière le rideau de fer attire de nouveau l'attention.

Deux universitaires portent un intérêt particulier à l'œuvre de Kiš : Guy Scarpetta de 1'Université de Reims et Jean Pierre Morel de l'Université Paris III. Tout au long des années 80 ils suivaient sont travail et continuent à l'étudier jusqu'à aujourd'hui. Jean Pierre Morel demeure l'un des passeurs culturels privilégié de la culture Kišienne en France (Keiflin et al., 2014). Il voit dans son œuvre une force particulière de médiation: C'est grâce à l'écrivain yougoslave qu'on doit la découverte de Musil et de Gombrovitch en France, (Ibid., 2014). 
C'est la rencontre avec les «nouveaux philosophes », Bernard-Henry Lévy et André Glucksmann dans les années 80 qui marque un nouveau pas dans la reconnaissance de Kiš à Paris. Les deux partagent l'estime particulière de l'écrivain, et la relation avec Henry-Lévy se transforme en véritable amitié. Kiš traduit le livre L'Éloge des intellectuels de celui-ci en serbe et Lévy souligne l'importance de l'écrivain yougoslave dans son projet des Derniers jours de Baudelaire. Cette interaction se révèle autant politique que culturelle. Bernard-Henri Lévy envisage à la fin des années 1980 de devenir l'éditeur principal de Danilo Kiš, chez Grasset (Keiflin et al., 2014).

En outre, la réception de son œuvre en France dépasse les frontières françaises, elle ouvre également des voies à la circulation de l'œuvre kišienne dans le monde - notamment aux États-Unis et en Scandinavie (la Suède précisément). Kiš lui-même mentionnait l'importance de la France comme médiatrice : «il faut passer par Paris pour exister» ( Kišs, 1995 : 105). C'est à partir du succès d'Un tombeau pour Boris Davidovitch en France que sort en 1980 une nouvelle édition américaine de ce livre (la première est publiée en 1978) avec la préface de Brodsky. Ainsi commence, sur le continent américain, la diffusion de la littérature de Kiš dont les amis très proches dans les années 1980 ne sont autres que Susan Sontag, Philip Roth, Brodsky lui-même.

Traduit en français, l'œuvre de Danilo Kiš a trouvé un nouveau public français et mondial, mais aussi des critiques qui suivaient et soutenaient l'auteur. Le contexte politique international dans lequel ses livres étaient publiés, surtout le Tombeau pour Boris Davidovitch (Paris, 1979), l'atmosphère encore chaud après la publication de l'Archipel Goulag de Soljenitsyne six ans plus tôt, ont contribué au vif intérêt pour son travail. La traduction française lui a permis cet accès aux centres, desquels parlait Pascal Casanova, «aux instances critiques et consécratrices ", la possibilité de montrer son œuvre à ceux qui décrètent ce qu'il faut lire. Loin d'être une simple naturalisation, la traduction en langue dominante a œuvrer pour «l'obtention d'un certificat de littérarité ». La réception française de cette œuvre nous a révélé les mécanismes d'un transfert culturel d'une langue dominée et le rôle de la foire aux enchères littéraire parisienne qui a permis à cette auteur la reconnaissance internationale. 
Pavle Sekeruš, Ivana Živančević Sekeruš, Virdjinija Popović

\section{PARIZ JE BERZA ZA KNJIŽEVNOST}

\section{Danilo Kiš kao kulturni posrednik između Francuske i Srbije}

\section{Rezime}

Danilo Kiš je u kontaktu sa francuskom kulturom od detinjstva. Francuski je naučio u školi, a prve prevode sa francuskog počeo je da objavljuje tokom studija u Beogradu. Angažovan kao lektor srpskohrvatskog jezika u Francuskoj, boravio je u Strazburu, Bordou i Lilu da bi se trajno nastanio u Parizu, gde je živeo do svoje smrti. Njegova najvažnija dela napisana u Francuskoj, prevedena i objavljena u najvećim pariskim izdavačkim kućama, ostaviće trag u francuskoj i svetskoj kulturi. Kombinujući teoriju kulturnih transfera Mišela Espanje i teorije o „svetskoj književnosti“ koje pretpostavljaju analizu transnacionalnih kulturnih prostora Paskal Kazanove pokzaćemo kako se Kiš svojom aktivnošću uspostavlja se kao centralna tačka razmene između dva kulturna prostora: francuskog, koji otvara vrata svetu i srpskom, jugoslovenskom i srednjoevropskom. Prvi mu nudi toleranciju kosmopolitskog grada i slobodu, ali i nepodnošljivu politizaciju i pretenzije svojstvene velikom centru, a drugi toplinu maternjeg jezika, bliskost i sigurnost, ali i provincijsku sredinu, uskogrudost, anarhiju i politički teror. On u Francuskoj nije pronašao samo odane kritičare, već i posvećenu publiku, iako njegovi romani nisu imali čitanost bestselera. Književnim posvećenjem u Francuskoj, Kišovo delo steklo je svetski prestiž.

Ključne reči: Danilo Kiš, kulturni transfer, Francuska, Srbija, prevodilac i autor

\section{REFERENCES BIBLIOGRAPHIQUES}

Casanova, P. (2002). Consécration et accumulation de capital littéraire. Actes de la recherche en sciences sociales, 144, 7-20. Consulté le 2 février 2019 https://www.persee.fr/doc/arss 0335-5322 2002 num $144 \quad 1 \quad 2804$

Janičić, P. - Lazić A. (1999). Podmuklo dejstvo biografije. Consulté le 5 mars 2019. http://www.kis.org.rs/web/Acitav/B/index.htm

Keiflin, E. - Marković, D. - Stanić,V. (2014/2). Danilo Kiš en France, «Exil », création, réception, 1935-1989, Bulletin de l'Institut Pierre Renouvin, 40, 95-109.

Kiš, D. (1979). Le tombeau de Boris Davidovitch. Paris : Gallimard.

Kiš, D. (1989). La Mansarde. Paris : Grasset.

Kiš, D. (1993). Paris, la grande cuisine des idées. In Homo poeticus. Paris : Fayard.

Kiš, D. (1995). Le résidu amer de l'expérience. Paris : Fayard.

Kiš, D. (2017). Psaume 44 suivi de la Mansarde. Paris : Fayard.

Matillon, J. (1980). Qu'est ce qu'un écrivain yougoslave à Paris ? La Quinzaine littéraire, 317. Consulté le 1 mars 2019 (https://serbica.u-bordeaux- 
montaigne.fr/index.php/revues/851-entretien-avec-danilo-kis-qu-est-ce-quun-ecrivain-yougoslave-a-paris-1980

Nikčević, T. (2014). Filip David-intervju. Peščanik. Consulté le 10 mars 2019 https://pescanik.net/filip-david-intervju/

Pavlović, J. (2016). Večnost Kišove Grobnice. Consulté le 3 novembre 2019 https://kaleidoskop-media.com/knji\%C5\%BEevnost/Danilo-Kis

Pivot, B. (2013). Danilo Kiš. Apostrophe. Consulté le 10 juin 2019 http://cavesdumajestic.canalblog.com/archives/2013/05/20/27141330.html

Srebro, M. (1980). Kiš, Danilo (1935-1989). Consulté le 15 juin 2019 https://serbica.u-bordeaux-montaigne.fr/index.php/k-91/177-ki-danilo$\underline{1935-1989}$

Srebro, M. (2005). "'Princ Danilo', vitez umetnosti i književnosti, i 'njeno visočanstvo' francuska kritika » [ Prince Danilo', chevalier de l'art et de la littérature et 'sa majesté' la critique française]. Letopis Matice srpske, 475, $5,753-781$.

Stojanović, B. (1999). Bibliografija Danila Kiša. 1999. Consulté le 5 septembre 2019 http://www.kis.org.rs/web/Bzivot/A/B/G/index.htm 